\title{
The role of sea-ice albedo in the climate of slowly rotating aquaplanets
}

\author{
Josiane Salameh $^{1,2}\left(\right.$ Max Popp $^{3} \cdot$ Jochem Marotzke $^{1}$
}

Received: 26 August 2016 / Accepted: 21 January 2017 / Published online: 25 February 2017

(C) The Author(s) 2017. This article is published with open access at Springerlink.com

\begin{abstract}
We investigate the influence of the rotation period $\left(\mathrm{P}_{r o t}\right)$ on the mean climate of an aquaplanet, with a focus on the role of sea-ice albedo. We perform aquaplanet simulations with the atmospheric general circulation model ECHAM6 for various rotation periods from one Earth-day to 365 Earth-days in which case the planet is synchronously rotating. The global-mean surface temperature decreases with increasing $\mathrm{P}_{r o t}$ and sea ice expands equatorwards. The cooling of the mean climate with increasing $\mathrm{P}_{r o t}$ is caused partly by the high surface albedo of sea ice on the dayside and partly by the high albedo of the deep convective clouds over the substellar region. The cooling caused by these deep convective clouds is weak for non-synchronous rotations compared to synchronous rotation. Sensitivity simulations with the sea-ice model switched off show that the global-mean surface temperature is up to $27 \mathrm{~K}$ higher than in our main simulations with sea ice and thus highlight the large influence of sea ice on the climate. We present the first estimates of the influence of the rotation period on the transition of an Earth-like climate to global glaciation. Our results suggest that global glaciation of planets with synchronous rotation occurs at substantially lower incoming solar irradiation than for planets with slow but non-synchronous rotation.
\end{abstract}

Keywords Non-synchronous - Rotation period · Aquaplanet $\cdot$ Albedo $\cdot$ Habitable zone

Josiane Salameh

josiane.salameh@mpimet.mpg.de

1 Max Planck Institute for Meteorology, Hamburg, Germany

2 International Max Planck Research School on Earth System Modelling, Hamburg, Germany

3 Princeton University, Princeton, NJ, USA

\section{Introduction}

A main target in planetary science is to identify the range of planetary and stellar parameters that are adequate to maintain a habitable climate. We use here the common definition of a habitable climate that liquid water is stable at the surface (Kasting et al. 1993). Important parameters influencing the habitability of a planet are its mass, its atmospheric composition and the intensity and the spectrum of the stellar flux it receives (Kasting et al. 1993; Kopparapu et al. 2014). Recently, it has been shown that furthermore the rotation period of a planet around its own axis influences its habitability (Yang et al. 2014). However, the influence of rotation on the climate is not well understood, especially if sea ice is taken into account.

The factors that constrained the Earth's current rotation period are thought to be a result of its particular past. Thus, similar potentially habitable planets around other stars may have a large range of rotation periods (Makarov et al. 2012; Makarov and Efroimsky 2013; Leconte et al. 2015). Moreover, the planetary rotation period is difficult to measure from the available observations of distant planets. Therefore, it is important to understand how long rotation periods (longer than the rotation period of Earth) affect the climate and thus the habitability of a planet.

Among slowly rotating terrestrial planets, those with a synchronous orbit have been extensively studied (Joshi 2003; Merlis and Schneider 2010; Edson et al. 2011; Abe et al. 2011; Yang et al. 2013; Leconte et al. 2013; Koll and Abbot 2015). In the case of synchronous rotation, the same side of the planet always faces the star, while the other side is in perpetual darkness. Slow but non-synchronous rotations have only been investigated either without changing the diurnal length with the rotation period (Hunt 1979; Del Genio and Zhou 1996; Navarra and Boccaletti 2002; Kaspi 
and Showman 2015) or without accounting for the effect of sea ice on the climate (Yang et al. 2014). The variation of the diurnal length is, however, quite important for the climate of a slowly rotating planet, because the day length can extend into many Earth months and cause a dayside-tonightside circulation to develop. In the absence of the effect of sea ice, clouds were found to have a strong cooling effect on the climate of slowly rotating Earth-like planets (Yang et al. 2014). However, the sea ice distribution is known to have a large impact on the climate due to the positive icealbedo feedback. Furthermore, we can infer from previous studies of synchronously rotating planets that the sea-ice distribution must change quite dramatically as the rotation period is increased from the present-day Earth rotation to a synchronous rotation. Preliminary results from a conference proceedings show that sea ice slightly varies with changing the rotation period in an aquaplanet with a mixed-layer ocean (Way et al. 2015). However, the distribution of sea ice and the mechanism by which sea ice affects the mean climate with changing rotation period is still unknown in the case of slow non-synchronous rotations.

The inner edge of the habitable zone of planets with long rotation periods was found to be closer to the star than that of planets with short rotation periods (Yang et al. 2014). However, no study has yet investigated the influence of the rotation period on the outer edge of the habitable zone, which is defined by the maximum greenhouse limit (Kasting et al. 1993; Kopparapu et al. 2013). This limit corresponds to the orbital distance where the surface is completely frozen in a $\mathrm{CO}_{2}$-rich atmosphere. A dense $\mathrm{CO}_{2}$ atmosphere is commonly assumed to be present near the outer edge of the habitable zone because the atmospheric $\mathrm{CO}_{2}$ concentration is expected to increase with decreasing temperature due to the slow-down of the carbonatesilicate cycle (Kasting et al. 1993). Here, we examine the orbital distance beyond which a global glaciation occurs at the surface for a fixed $\mathrm{CO}_{2}$ concentration in an Earth-like atmosphere.

To this end, we perform simulations of an aquaplanet across a large range of long rotation periods, taking both the sea ice and the variable diurnal length into account. The studied rotation periods range from one Earth-day to 365 Earth-days in which case the planet would be synchronously rotating. We use the state-of-the-art atmospheric general circulation model (GCM) ECHAM6 (Stevens et al. 2013) in an aquaplanet setup in perpetual equinox conditions. The model and the slowly rotating aquaplanet configuration are briefly described in Sect. 2. In Sect. 3, we show how sea ice affects the climate and compare the mean states of our main simulations and our sensitivity simulations with the sea-ice model switched off. We show how the temperature gradients change from a zonally uniform pattern to a dayside-to-nightside pattern as the rotation period increases (Sect. 4) and how this change affects the contribution of the sea-ice albedo to the planetary albedo (Sect. 5). In Sect. 6, we explain the diurnal variations in the atmosphere, in particular the cloud radiative effects as a function of rotation period. The implications of the rotation for the point of global glaciation are discussed in Sect. 7. A discussion of the results is presented in Sect. 8 and a conclusion in Sect. 9.

\section{Model and simulations setup}

Our 3D aquaplanet simulations are performed with the GCM ECHAM6, revision 6.3.0 (Stevens et al. 2013). The atmosphere is coupled to a static $50 \mathrm{~m}$ deep mixed-layer ocean without prescribing the convergence of an oceanic heat transport at the surface (no q-flux). The primitive equations of the dynamical core are discretised following the spectral-transform method. The model resolution used is T31L47, which is equivalent to a horizontal resolution of $3.75^{\circ}$, and consists of 47 vertical hybrid sigma-pressure levels up to a pressure of $0.01 \mathrm{hPa}$. The radiative transfer of the shortwave and longwave fluxes is based on the Rapid Radiative Transfer Model (Mlawer et al. 1997; Iacono et al. 2008). Moist convection is parameterised according to the mass-flux scheme by Tiedtke (1989) with modifications to the penetrative convection according to Nordeng (1994). We employ a diagnostic cloud-cover scheme based on relative humidity (Sundqvist et al. 1989) and the micro-physical scheme introduced in Lohmann and Roeckner (1996), which includes prognostic equations for cloud water and ice. For a more detailed description of the model we refer to Stevens et al. (2013).

The thermodynamics of the sea ice are represented by the zero-layer Semtner model (Semtner 1976), which computes the sea-ice temperature and thickness from the energy balance at the surface. Sea ice forms when the diagnosed temperature is below the freezing temperature of sea water, which is fixed at $-1.8^{\circ} \mathrm{C}$. A grid cell is either open-water with a sea-ice concentration of zero, or completely seaice covered with a concentration of 1 . A fractional sea-ice cover of a grid cell is not included in this setup. Snow is only allowed to accumulate over grid cells that are covered with sea ice. The computation of surface albedo distinguishes between open water, bare sea ice and snow-covered sea ice. The albedo of an open-water grid cell consists of a direct and a diffuse contribution from both the visible and the near-infrared part of the solar spectrum. Only the direct component of the albedo depends on the zenith angle. The albedo of a bare sea-ice surface is a linear function of the surface temperature with a minimum of 0.55 at $0{ }^{\circ} \mathrm{C}$ and a maximum of 0.75 for a temperature below $-1{ }^{\circ} \mathrm{C}$. Snow has an albedo minimum of 0.65 and a maximum of 0.8 . 
To facilitate the investigation of the influence of the rotation period on the climate, the seasonal variability is neglected by setting the obliquity and eccentricity to zero. The orbital period $\mathrm{P}_{\text {orb }}$, which is the period for a planet to complete one revolution around its star, is fixed to 365 Earth-days. The rotation period $\mathrm{P}_{r o t}$ refers to the time required for a planet to complete one full revolution around its axis of rotation. By modifying $\mathrm{P}_{r o t}$ of a planet in a circular orbit, the day length $\mathrm{P}_{d a y}$-the equivalent time of one complete day and night cycle - varies as a combination of the orbital and rotational periods as:

$P_{\text {day }}=\frac{1}{P_{\text {rot }}^{-1}-P_{\text {orb }}^{-1}}$

Note that the day length exceeds the specified value of $\mathrm{P}_{\text {rot }}$ in all the considered cases. A day lasts longer than one orbital period if $\mathrm{P}_{r o t}$ is greater than $\mathrm{P}_{\text {orb }} / 2$.

We modify the zenith angle to be a function of $\mathrm{P}_{d a y}$ such that the corresponding incoming shortwave radiation at the top of the atmosphere is represented consistently. The incoming solar radiation in our default simulations corresponds to the spectrum of the Sun with a total solar constant of $1361 \mathrm{~W} / \mathrm{m}^{2}$. In all simulations, the $\mathrm{CO}_{2}$ concentration is fixed at $800 \mathrm{ppmv}, \mathrm{CH}_{4}=0, \mathrm{~N}_{2} \mathrm{O}$ is equal to its Earth-like value of $309.5 \mathrm{ppb}$, and the ozone is prescribed to a meridionally symmetric and zonally uniform climatology. The reference simulation has a rotation period of 1 Earth-day, and the other simulations have rotation periods that are multiples of one Earth day. The rotation periods considered are 1, 4, 8, 16, 32, 64, 128, 182, 200, 256, 300 and 365 Earth-days. Each simulation is integrated for 30 years after equilibrium is reached, in order to compute the steady-state statistics. We perform a set of sensitivity experiments where the sea-ice model is turned off with the same rotation periods. In this case, the water is allowed to cool below the freezing temperature, but without forming a sea-ice layer. We will henceforth refer to our main simulations with sea ice turned on as iceON and the ones with sea ice turned off as iceOFF. We perform additional experiments with reduced solar irradiance to assess the maximum solar irradiance at which global glaciation occurs for each rotation period.

\section{Mean climate with long rotation periods}

We start by describing how the mean climate responds to a slowing down in rotation in our main iceON simulations. Increasing the rotation period leads to a monotonic decrease in the global-mean surface temperature (GMST) of the aquaplanet (Fig. 1). The largest drops in GMST of more than $10 \mathrm{~K}$ occur between $\mathrm{P}_{r o t}=32$ and $\mathrm{P}_{r o t}=64$ Earthdays, and between $\mathrm{P}_{r o t}=182$ and $\mathrm{P}_{r o t}=200$ Earth-days

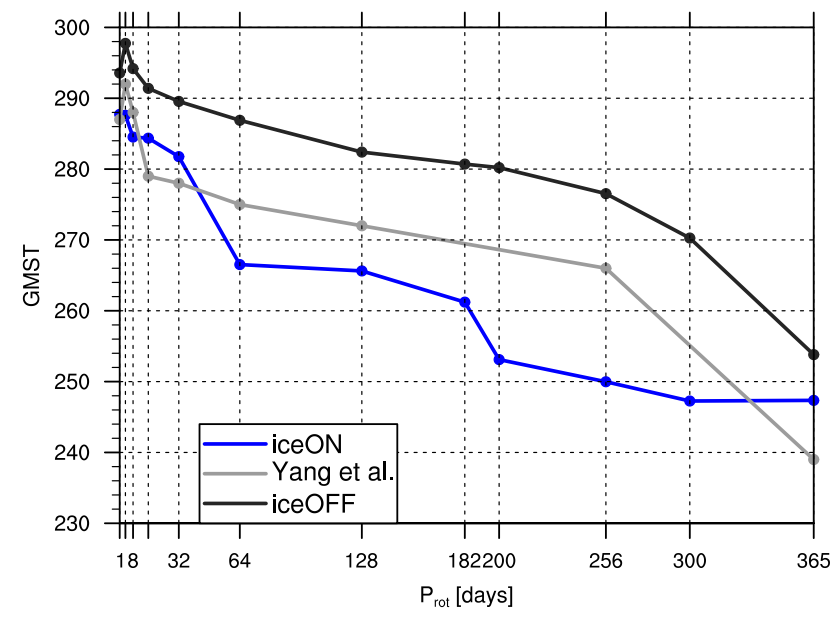

Fig. 1 Global and temporal mean over the last 30 years of simulation of the surface temperature $(\mathrm{K})$ as a function of the rotation period $\mathrm{P}_{r o t}$. The GMST of our simulations with the sea-ice model switched on (iceON) are shown in blue, and without the sea-ice model (iceOFF) in black. For comparison, the GMST of (Yang et al. 2014) is shown in grey

(see Sect. 4 for partial explanation). Consistent with the decrease in GMST, the global-mean fraction of sea ice increases with $\mathrm{P}_{\text {rot }}$ up to a maximum of $75 \%$ at $\mathrm{P}_{\text {rot }}=300$ Earth-days (Fig. 2b). However, the global-mean fraction of sea ice decreases again by $5 \%$ between $\mathrm{P}_{\text {rot }}=300$ and 365 Earth-days. We compute the temporal and global mean of the "effective surface albedo", which is the temporal and global mean of the reflected shortwave radiation at the surface divided by the temporal and global mean incoming shortwave radiation at the surface, in order to analyze the influence of sea ice on the surface energy balance. The global mean of the effective surface albedo $\left(\alpha_{S}\right)$ increases with $\mathrm{P}_{r o t}$ up to 0.46 at 200 Earth-days, then decreases for even longer rotation periods, even though the global-mean fraction of sea ice increases further with increasing rotation period (Fig. 2b). This occurs because only the dayside contributes to the effective surface albedo and because the sea-ice cover on the dayside starts to decrease with increasing rotation period for $\mathrm{P}_{r o t}$ longer than 200 Earth-days. The global-mean planetary albedo $\left(\alpha_{P}\right)$ diagnosed at the top of the atmosphere increases with $\mathrm{P}_{r o t}$ up to $\mathrm{P}_{r o t}=200$ Earthdays and then remains constant at a value of 0.46 for larger values of $\mathrm{P}_{\text {rot }}$ (Fig. 2a).

To investigate the influence of sea ice on the mean state for the different rotation periods, we compare the global-mean climates of our main simulations iceON and of our sensitivity simulations iceOFF. The difference in the GMST between iceON and iceOFF remains at around $6 \mathrm{~K}$ for rotation periods up to 32 Earth-days, then increases to $27 \mathrm{~K}$ as the rotation period is increased from 32 to 64 Earth-days. The difference then remains close 

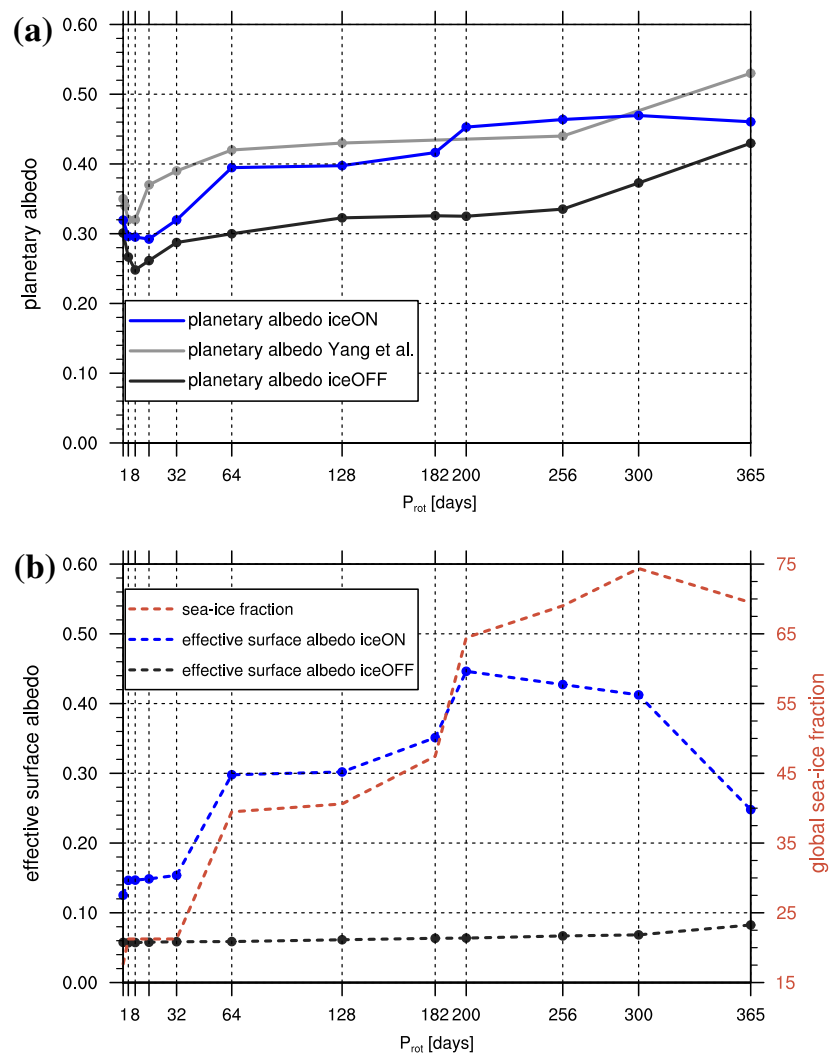

Fig. 2 Global and temporal mean over the last 30 years of simulation $\mathbf{a}$ of the planetary albedo and $\mathbf{b}$ of the effective surface albedo and the sea-ice fraction ( $y$-axis on the right) as a function of the rotation period $\mathrm{P}_{r o t}$. The simulations with the sea-ice model switched on are in blue (iceON) and the ones with the sea-ice model switched off are in black (iceOFF). The $\alpha_{P}$ values of Yang et al. (2014) are in grey

to $27 \mathrm{~K}$ for periods of up to 300 Earth-days and drops back to $6 \mathrm{~K}$ in the synchronously rotating case. In iceOFF simulations, the effective surface albedo (Fig. 2b) follows closely the value of the ocean albedo of 0.07 , with slight deviations due to scattering and absorption of solar radiation in the atmosphere. Similarly to the GMST, large differences in the global-mean planetary albedo (up to 0.15 ) between iceON and iceOFF simulations occur for the same range of $\mathrm{P}_{r o t}$ between 64 and 300 Earth-days (Fig. 2a).

These large differences in the GMST and global-mean planetary albedo between iceON and iceOFF highlight the importance of accounting for sea ice when simulating long rotation periods. To understand the mechanisms that drive the changes in climate with changing the rotation period, we now analyze the diurnal variations of the relevant quantities.

\section{The diurnal contrast at the surface}

Due to the lack of zonal asymmetries in an aquaplanet configuration, the large-scale circulation is typically analysed from temporal and zonal means of the fields of interest. This analysis does not remain adequate for rotation periods longer than 32 Earth-days, because then the zonal differences caused by the strong differential heating become more and more pronounced. The diurnal cycle of the present-day Earth climate can be analysed by performing a Fourier series of desired quantities. The magnitude and phase of these quantities are thus obtained (Bechtold et al. 2014). This method could also be applied to long rotation periods, but we choose to analyse the day-to-night characteristics at different $\mathrm{P}_{r o t}$ by computing temporal averages with respect to the time of day. For each $\mathrm{P}_{r o t}$, the day has a different length and is obtained by solving equation (1). The time of day is defined based on the angle between the longitude of a given location and the longitude of the substellar point. We use the convention here that $0^{\circ}$ is sunset, $90^{\circ} \mathrm{W}$ is noon, $180^{\circ} \mathrm{E}$ is dawn and $90^{\circ} \mathrm{E}$ is midnight. We use this method to visualize the diurnal cycle of quantities in Figs. 3, 4, 5 and 8. Previous studies of non-synchronous rotations rather presented snapshots than applying similar time-averages.

The day-to-night contrast in surface temperature becomes apparent at a rotation period of 64 Earth-days, while the day-to-night contrast in the sea-ice distribution appears at $\mathrm{P}_{\text {rot }}=182$ Earth-days (Fig. 3). The first large drop in the GMST (by $15 \mathrm{~K}$ ) between a $\mathrm{P}_{\text {rot }}$ of 32 and 64 Earth-days (Fig. 1) is related to the equatorward expansion of sea ice from latitude $52^{\circ}$ to $36^{\circ}$ in each hemisphere and to a nearly $10 \mathrm{~K}$ cooling within the tropical band that stretches from latitude $30^{\circ} \mathrm{S}$ to $30^{\circ} \mathrm{N}$. It is as well associated with the transition in the dynamical regime from a meridional atmospheric circulation to a "Walker cell" regime (day-to-night circulation) (Showman et al. 2013), where the subtropical jets disappear at $\mathrm{P}_{\text {rot }}=64$ Earth-days. Note that the Rossby number becomes large with increasing rotation period, and the horizontal motion becomes isotropic because the geostrophic balance breaks down (Merlis and Schneider 2010; Showman et al. 2013). The second large drop in the GMST (by $10 \mathrm{~K}$ ) between simulations with a $\mathrm{P}_{r o t}$ of 182 and 200 Earth-days (Fig. 1) occurs when sea ice forms over the equator during nighttime. The sea ice then persists substantially into the daytime before melting in the late morning. This occurs in the simulations with $\mathrm{P}_{r o t}$ of 200, 256 and 300 Earth-days.

The response of ice-free and ice-covered surfaces to the heating by solar radiation on the dayside and to the cooling on the nightside has different time-scales. This is especially apparent in the simulations with $\mathrm{P}_{r o t}$ between 64 and 300 Earth-days. Over ice-free surfaces, 
Fig. 3 Temporal mean over the last 30 years of simulations with respect to the time of the day. The surface temperature $(\mathrm{K})$ is denoted by colour filled contours and the ice line by a black contour line. Each panel corresponds to one rotation period from the 12 simulations. These are the panels from top left to bottom right: $\mathrm{P}_{\text {rot }}=1,4,8,16$, $32,64,128,182,200,256,300$ and 365 (Earth-days)
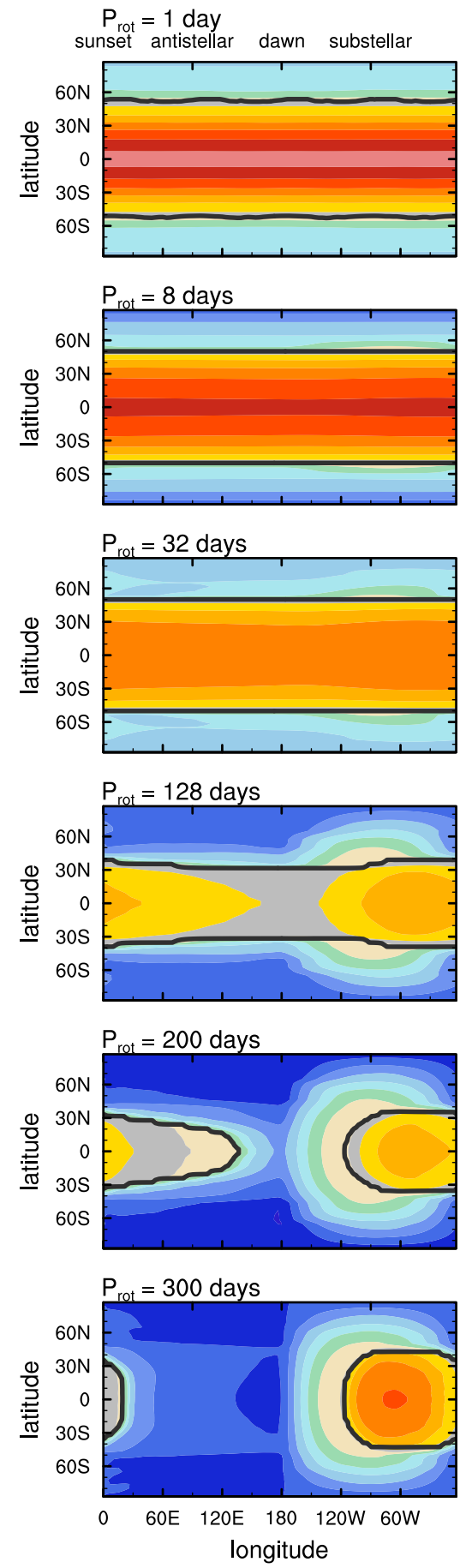
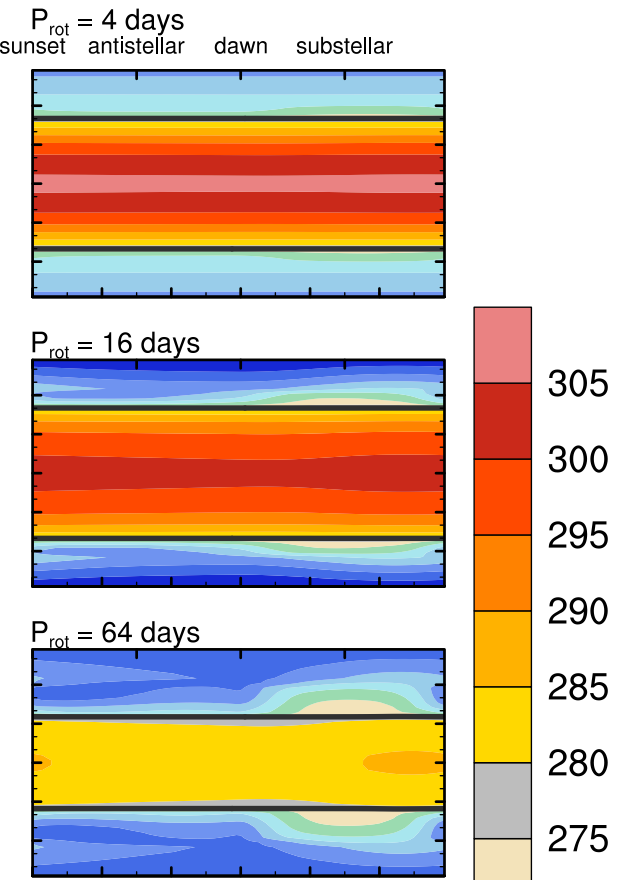

290

285

280

275

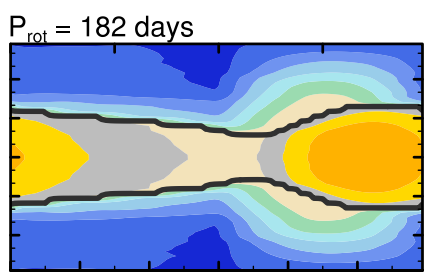

270

260

250

240

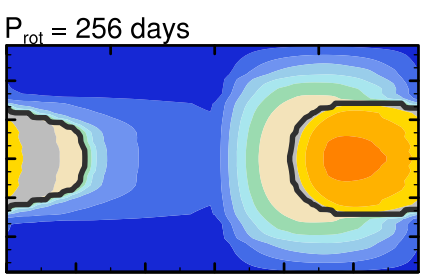

230

220

210 the response of the SST to the energy from the incoming solar radiation is slow due to the large thermal inertia of the mixed-layer ocean. Thus, along the ice-free equatorial band, the highest surface temperature is shifted towards the east of the substellar meridian at $90^{\circ} \mathrm{W}$ - towards the afternoon time (Fig. 3). After crossing the sunset meridian at $0^{\circ} \mathrm{W}$, the surface temperature gradually decreases during the long nights and attains its minimum near dawn. Temperatures over surfaces covered with sea ice respond faster to the incoming solar radiation than over open-water surfaces, because sea ice has a lower thermal inertia than the ocean below. For the same reason, the temperatures over sea ice drop more quickly on the nightside. 
Fig. 4 Temporal mean over the last 30 years of simulations with respect to the time of the day of the planetary albedo on the dayside. The blue crossed pattern corresponds to a cloud fraction larger than 0.6 at the $500 \mathrm{hPa}$ vertical pressure level. Each panel corresponds to one rotation period from the 12 simulations. These are the panels from top left to bottom right, $\mathrm{P}_{r o t}=1,4,8,16,32,64$, $128,182,200,256,300$ and 365 (Earth-days)
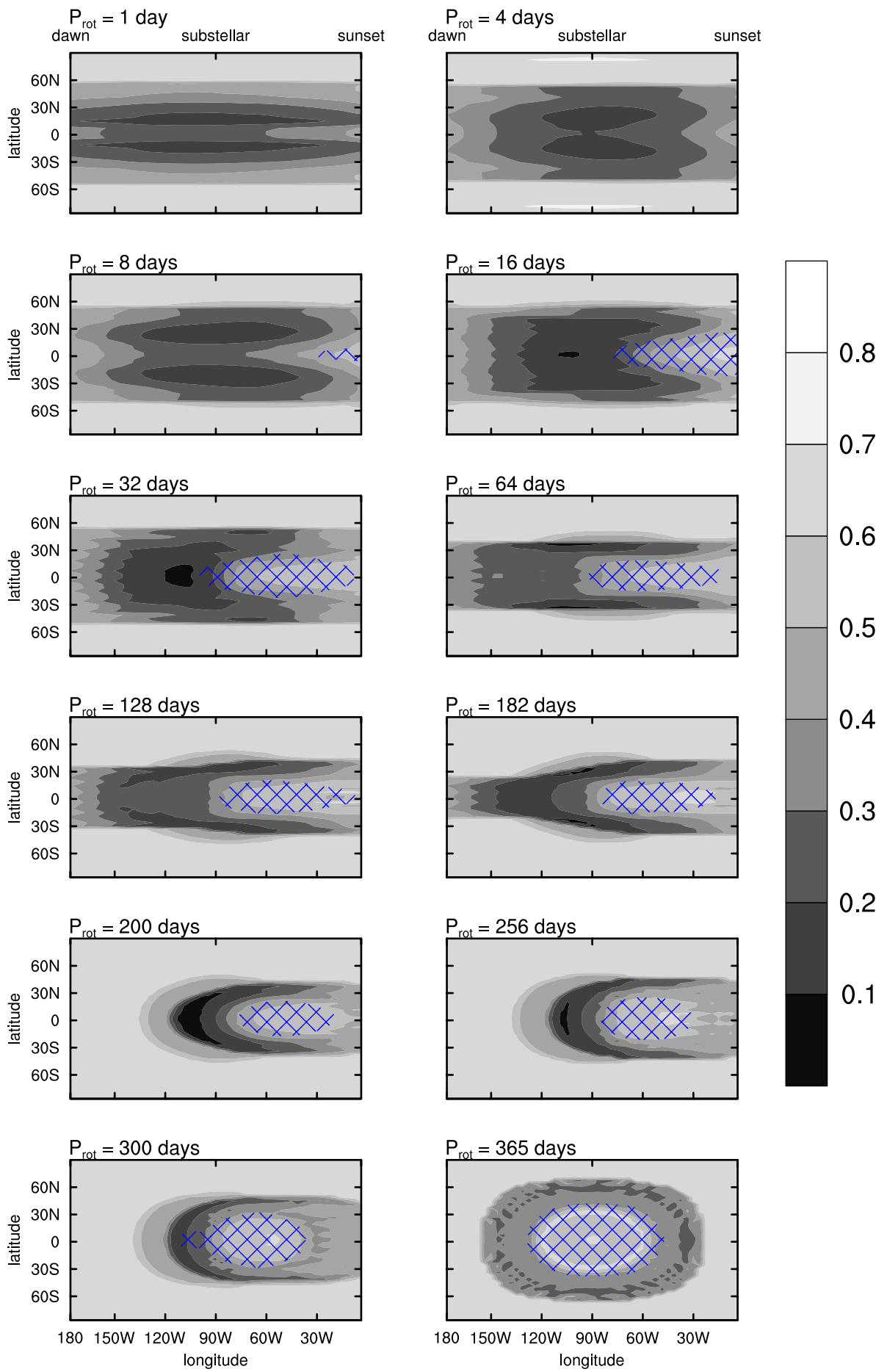

\section{Surface contribution to planetary albedo}

Under present-day Earth climate, the surface albedo has only a small influence on the planetary albedo compared to the atmospheric albedo (Donohoe and Battisti 2011). Regardless of the type of the Earth's surface and thus its surface albedo, Donohoe and Battisti (2011) found that up to $88 \%$ of the planetary albedo comes from the atmospheric contribution. The atmospheric contribution consists of the cloud albedo, scattering by air and aerosols, and absorption by water vapour.

Following Donohoe and Battisti (2011), we quantify the different contributions to the planetary albedo for each slowly-rotating-aquaplanet simulation (Figs. 4, 5). The total planetary albedo $\alpha_{P}$ is the sum of a surface contribution $\alpha_{S, P}$ and an atmospheric contribution $\alpha_{A, P} . \alpha_{S, P}$ and $\alpha_{A, P}$ are 
Fig. 5 Temporal mean over the last 30 years of simulations with respect to the time of the day of the relative surface contribution to the planetary albedo. Since the total planetary albedo is the sum of its surface and atmospheric contribution, the red colour scale denote a surface contribution larger than $50 \%$, while the grey colour scale refers to an atmospheric contribution larger than $50 \%$. Each panel corresponds to one rotation period from the 12 simulations. These are from $t o p$ left to bottom right, $\mathrm{P}_{\text {rot }}=1$, 4, 8, 16, 32, 64, 128, 182, 200, 256, 300 and 365 (Earth-days)
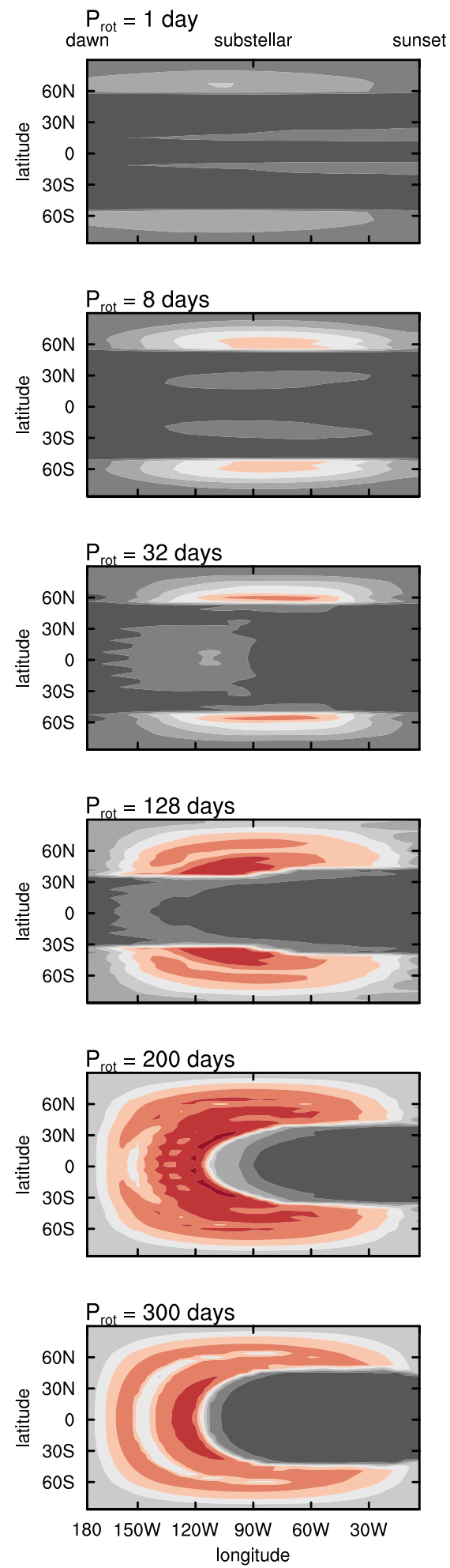
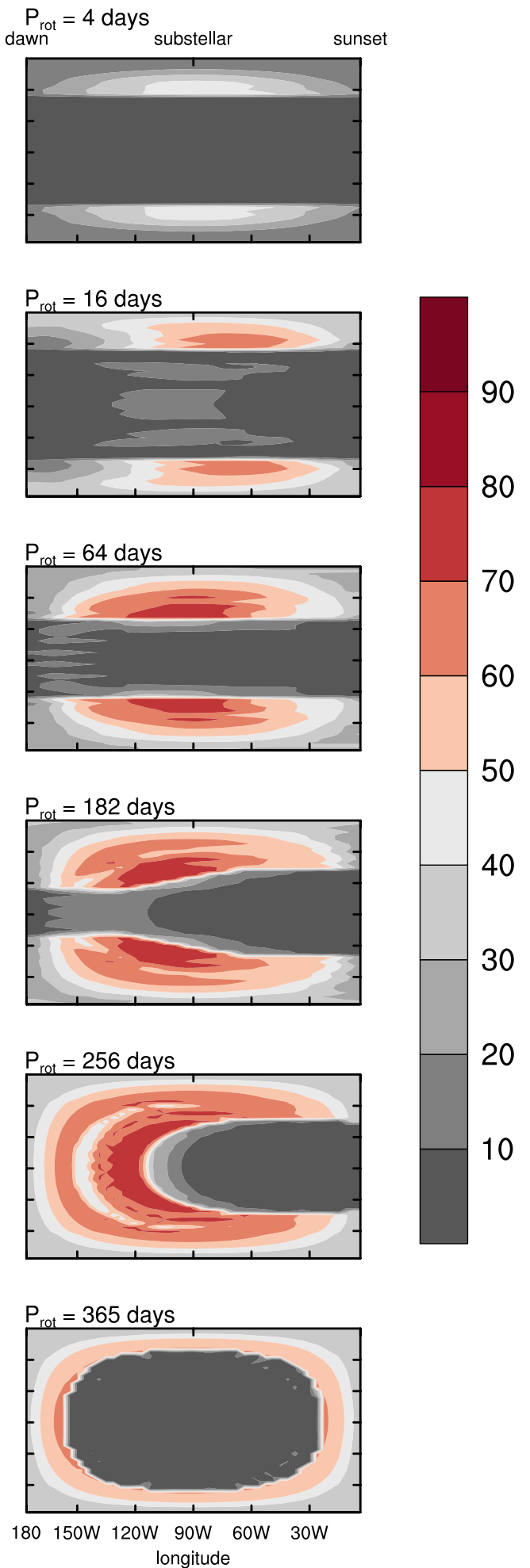

calculated based on the single-layer solar-radiation model introduced in Donohoe and Battisti (2011). Note that only the dayside is shown in Figs. 4 and 5, because the albedo does not influence the energetics on the nightside.

For rotation periods up to 32 Earth-days, the pattern of $\alpha_{P}$ is zonally relatively uniform, with a maximum of 0.7 at high latitudes and values as low as 0.2 at lower latitudes especially in the dry subtropics (Fig. 4). As the rotation period increases, zonal variations in $\alpha_{P}$ increase. The areas with high $\alpha_{P}$ coincide with either the location of surfaces covered with sea ice or with the location of optically thick convective clouds. High values of planetary albedo 
associated with deep convective clouds were already discussed in the case of synchronous rotation (Yang et al. 2013) and in the case of rotation periods longer than 100 Earth-days (Yang et al. 2014). However, the impact of the ice albedo on the energy balance of slowly rotating planets has not yet been investigated.

For periods longer than 32 Earth-days, the ratio of $\alpha_{S, P}$ to $\alpha_{P}$ takes values of up to $80 \%$ over regions with a high surface albedo on the dayside (Fig. 5). The contribution of the surface to the planetary albedo is particularly large for simulations with $\mathrm{P}_{\text {rot }}=200,256$ and 300 Earth-days, in which sea ice has spread along the equator during the morning time. Thus, for slow non-synchronous rotations, the surface contribution dominates westward and poleward from the substellar point, where sea ice is present at the surface. A large atmospheric contribution occurs in the area of high cloud cover east of the substellar point, and at low zenith angle where atmospheric scattering contributes strongly to the planetary albedo. For slow non-synchronous rotations, the highly reflective surface plays an important role in cooling the climate. Note that even with a high surface albedo, the atmospheric contribution to the planetary albedo would dominate if clouds were present over sea ice. However, this is not the case for most sea-ice covered areas that are exposed to solar radiation.

In the simulation with synchronous rotation $\left(\mathrm{P}_{\text {rot }}=365\right.$ Earth-days), the deep convective clouds cover a large fraction of the dayside. This leads to a large atmospheric contribution to the planetary albedo (larger than 90\%; Fig. 5). The sea-ice albedo contributes only near the edge of the day terminator, and even there only moderately (40-60\%). The global sea-ice coverage is high in this simulation, but since most of the sea ice is located on the nightside, only $25 \%$ of the incident solar radiation at the surface is reflected (Fig. 2b).

\section{Diurnal variations in the atmosphere}

\subsection{Cloud radiative effects}

Although deep convective clouds typical of the substellar region in synchronously rotating planets occur also in non-synchronous rotations, the magnitude of their cloud-radiative effects (CRE) differs in the two cases. The shortwave and longwave CRE are defined as the difference between the full-sky and clear-sky shortwave and longwave fluxes respectively, at the top of the atmosphere (TOA) (Ramanathan and Inamdar 2006). The global- and time-mean shortwave CRE for non-synchronous rotations ranges between $-62 \mathrm{~W} / \mathrm{m}^{2}$ for $\mathrm{P}_{r o t}=1$ Earth-day and $-36 \mathrm{~W} / \mathrm{m}^{2}$ for $\mathrm{P}_{r o t}=200$ Earth-days. For rotation periods longer than $\mathrm{P}_{r o t}=200$ Earth-days, the shortwave
CRE gradually increases with $\mathrm{P}_{r o t}$, and the strongest cooling occurs for the synchronous case where the shortwave CRE has a magnitude of $-100 \mathrm{~W} / \mathrm{m}^{2}$ (Fig. 6). In contrast, the global and time-mean longwave CRE is nearly constant with $\mathrm{P}_{r o t}$ and small in comparison to the magnitude of the shortwave CRE. Therefore, clouds cool the climate at any rotation period. But the cooling imposed by clouds is larger in the simulation with synchronous rotation than in the simulations with non-synchronous rotations. We now explain what causes these differences in CREs with different rotation.

\subsection{The large-scale circulation}

Increasing the rotation period modifies the large-scale circulation from a nearly zonally uniform pattern for rotation periods shorter than 32 Earth-days to a substellar-antistellar pattern for rotation periods longer than 32 Earth-days. The substellar-antistellar circulation is characterized by a strong convergence of near-surface winds over the substellar region with high surface temperature and a strong upward motion (not shown). At higher vertical levels, the winds diverge, and downward motion spreads over the rest of the domain. This large-scale motion is driven by a combination of low-level moisture convergence and tropospheric stability. However, the strength of the vertical motion and the atmospheric profile vary with rotation.

To understand the variations of the strength and distribution of the large-scale vertical motion with the

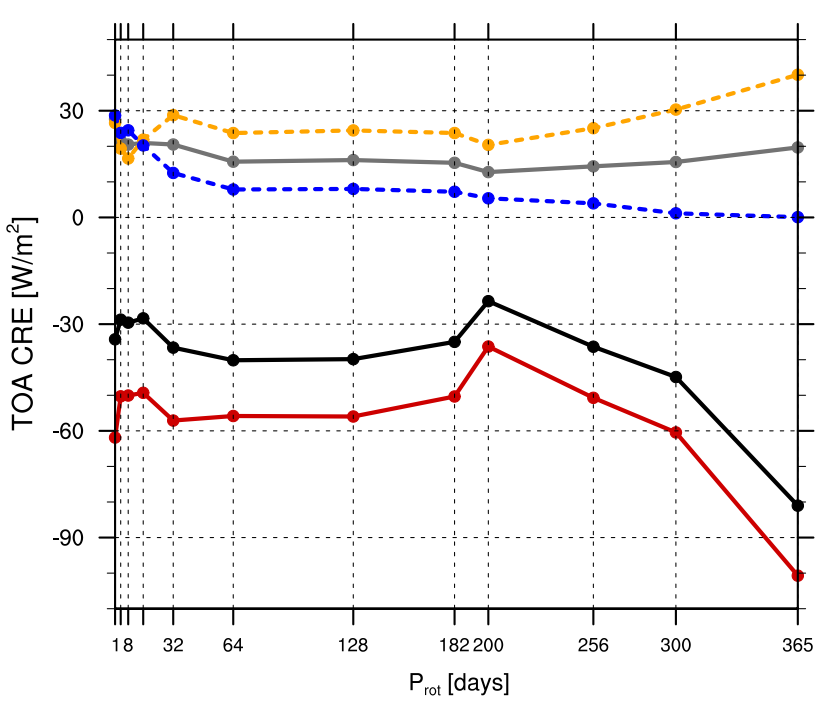

Fig. 6 Global and temporal average over the last 30 years of simulation of the shortwave CRE (solid red), longwave CRE (solid grey) and the total CRE (solid black) as a function of $\mathrm{P}_{r o t}$. The longwave CRE averaged over the dayside (dashed yellow) and averaged over the night side (dashed blue) 
rotation period over the substellar region, we compute the probability density function (PDF) of the upward vertical velocity at a pressure level of $500 \mathrm{hPa}\left(\omega_{500}\right)$ over the hot-spot region, similarly to Bony et al. (2004). The hot-spot region is defined as the area with a surface temperature equal to $280 \mathrm{~K}$ or above within the tropics and with a negative vertical velocity. A negative value of $\omega_{500}$ indicates an upward motion while a positive value indicates a downward motion. Note that a convective event is considered deep when $\omega_{500}$ is less than $-50 \mathrm{hPa} /$ day.

By increasing the rotation period from $\mathrm{P}_{r o t}=1$ to 32 Earth-days, the tail of the PDF expands and extends further towards high negative values of $\omega_{500}$, and the median value of the PDF shifts towards high negative values of $\omega_{500}$ (Fig. 7a). This indicates that deep convective events become more frequent and more intense. Deep convective events strengthen further with increasing $\mathrm{P}_{r o t}$ until $\mathrm{P}_{r o t}=200$ Earth-days (Fig. 7b). The magnitude of the very strong convective events decreases again with increasing $\mathrm{P}_{\text {rot }}$ beyond 200 Earth-days. For rotation periods longer than 64 Earth-days, 50\% of the upward motion occurs during deep convective events over the hot-spot, because the medians of their PDF are below $\omega_{500}=-100 \mathrm{hPa} /$ day. However, weak subsidence is the most frequent vertical motion over the hot-spot region for all rotation periods, because the time-mean area with an upward motion over the hot-spot is less than $50 \%$ of the tropical area for any $\mathrm{P}_{r o t}$ (Table 1). For each slow rotation, these very strong upward motions over the hot-spot area promote the formation of deep convective clouds (Fig. 8).

\subsection{Atmospheric profile over the dayside}

The upward motions transport latent heat and moisture to higher levels, leading to higher tropospheric temperature and more water vapour over that area than elsewhere. This is illustrated by a tilt in contours of constant temperature and specific humidity in the lower troposphere between day and night (Fig. 8). This tilt becomes more pronounced for longer rotation periods. With $\mathrm{P}_{r o t}$ increasing from 1 to 200 Earth-days, the area with high surface temperature over the dayside shrinks, and the lower-troposphere temperature and specific humidity decrease (Figs. 3, 8). They both increase again with $\mathrm{P}_{r o t}$ for $\mathrm{P}_{r o t}$ longer than 200 Earth-days.

The difference between the vertical profiles of the dry static energy (DSE) and the moist static energy (MSE) is proportional to the amount of water vapour. At the substellar point, the differences between the MSE and DSE is smallest for $\mathrm{P}_{r o t}=200$ Earth-days and largest for $\mathrm{P}_{r o t}=365$ Earth-days (Fig. 9). The mid- to upper troposphere is close to saturation in the region of the hot-spot for all these simulations. This is illustrated by an overlap of the MSE and the saturated MSE. This may explain why the cloud cover is high in this region, because it is also consistent with the frequent occurrence of convection over the hot-spot location. Similarly to water vapour, the liquid- and ice-water paths over the dayside decrease with $\mathrm{P}_{r o t}$ until $\mathrm{P}_{r o t}=200$ Earthdays, then increase for longer rotation periods (Figs. 8, 10). The variations of water clouds with $\mathrm{P}_{r o t}$ over the dayside are more pronounced in comparison to ice clouds.

These variations of the distribution of the deep convective clouds with $\mathrm{P}_{r o t}$ explain the change in magnitude of the shortwave CRE in the afternoon, which decreases with $\mathrm{P}_{r o t}$ until $\mathrm{P}_{r o t}=200$ Earth-days and then increases again
Fig. 7 The probability density function (\%) of the upward vertical velocity at $500 \mathrm{hPa}$ pressure over the hot-spot region (the region with surface temperature higher than 280 K) $\mathbf{a}$ for $\mathrm{P}_{r o t}=1,4,8,16,32$ (Earth-days) and $\mathbf{b}$ for $\mathrm{P}_{r o t}=64$, $128,182,200,256,300,365$ (Earth-days). The median of the PDF of each $\mathrm{P}_{\text {rot }}$ is shown in crossed circle
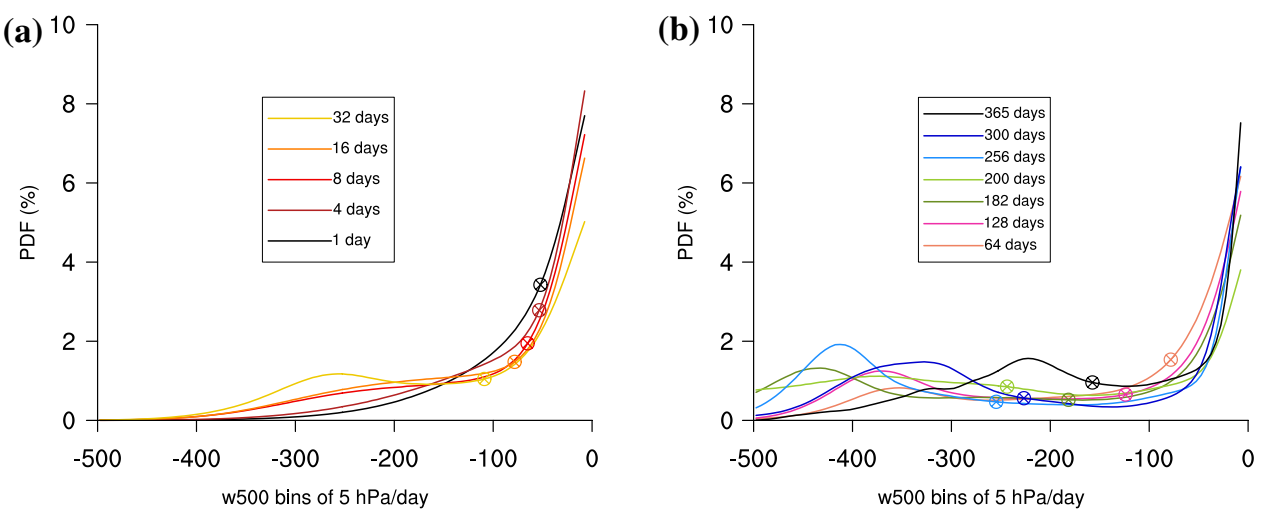

Table 1 The time-mean area with an upward motion $\left(\omega_{500}<0\right)$ over the hot-spot $(\mathrm{SST} \geq 280 \mathrm{~K})$ as a fraction of the tropical area

\begin{tabular}{|c|c|c|c|c|c|c|c|c|c|c|c|c|}
\hline $\mathrm{P}_{r o t}$ (Earth-days) & 1 & 4 & 8 & 16 & 32 & 64 & 128 & 182 & 200 & 256 & 300 & 365 \\
\hline Area with upward motion over hot-spot (\%) & 44 & 45 & 44 & 45 & 46 & 45 & 33 & 23 & 18.5 & 20 & 22 & 20.5 \\
\hline
\end{tabular}



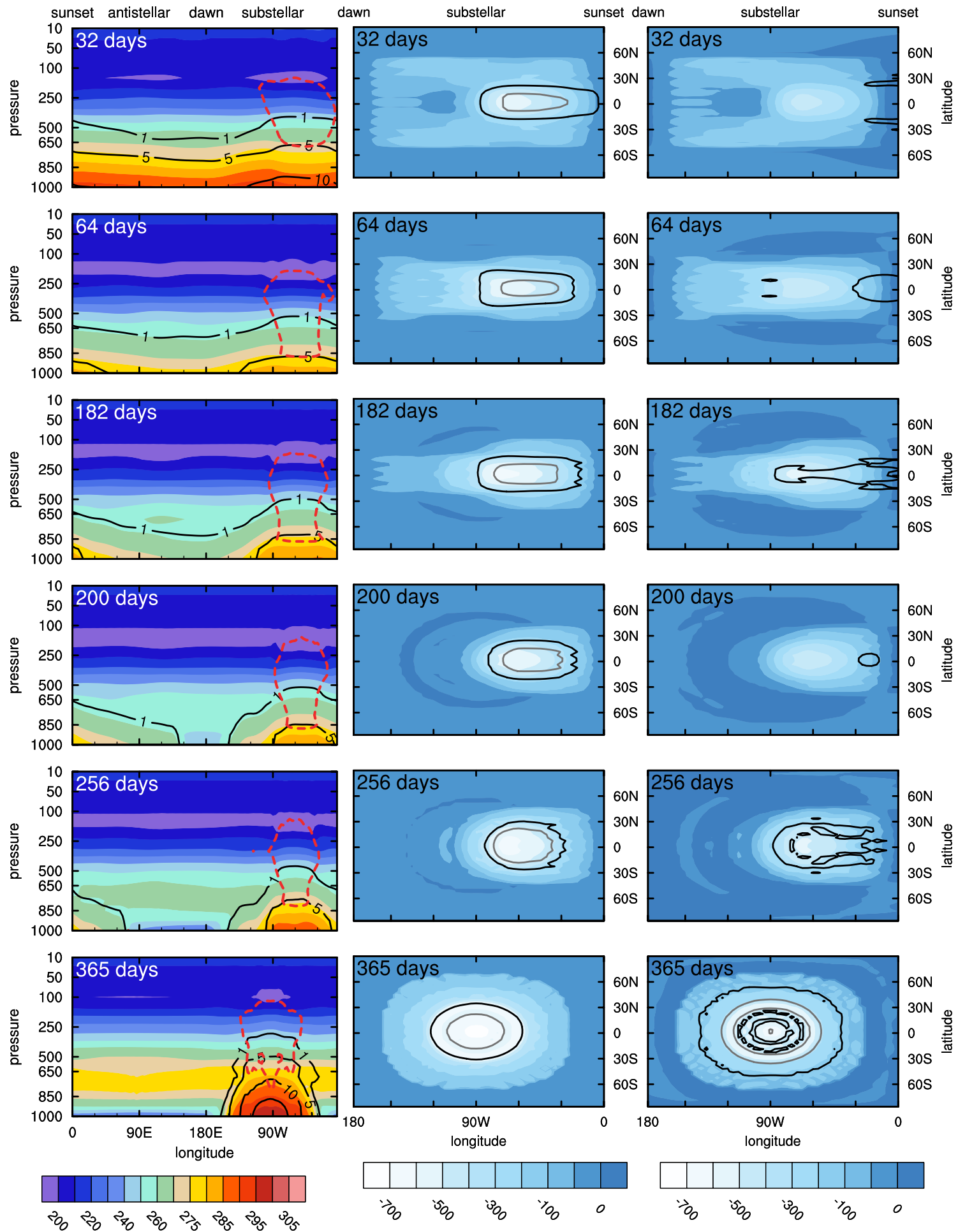

Fig. 8 Temporal mean over the last 30 years of simulation with respect to the time of the day. First column A vertical cross-section along the equator of the temperature $(\mathrm{K})$ (coloured contours), the specific humidity $(\mathrm{g} / \mathrm{kg})$ (black contour lines) and the cloud fraction equal to 0.6 (dashed red line), highlighting the thick convective clouds over the substellar region. Second column Shortwave CRE (W/ $\mathrm{m}^{2}$ ) over the dayside (filled blue contours). The vertically-integrated ice clouds are denoted by contour lines (only for $0.1 \mathrm{~kg} / \mathrm{m}^{2}$ in black and $0.2 \mathrm{~kg} / \mathrm{m}^{2}$ in grey). Third column Total CRE $\left(\mathrm{W} / \mathrm{m}^{2}\right)$ over the dayside (filled blue contours). The vertically-integrated water clouds are denoted by contour lines $\left(0.1 \mathrm{~kg} / \mathrm{m}^{2}\right.$ in black and $0.3 \mathrm{~kg} / \mathrm{m}^{2}$ in grey) 
Fig. 9 The vertical profiles of dry static energy (black), moist static energy (blue) and saturated moist static energy (red) $(\mathrm{KJ} / \mathrm{kg})$ at the warmest point (solid lines) and the antistellar point (dash lines) at the equator. Only four $\mathrm{P}_{r o t}$ are shown: 182, 200, 256 and 365 (Earth-days). For $\mathrm{P}_{r o t}=256$ and 365 Earth-days, the DSE line at the antistellar point is masked by the MSE line because the atmosphere is dry
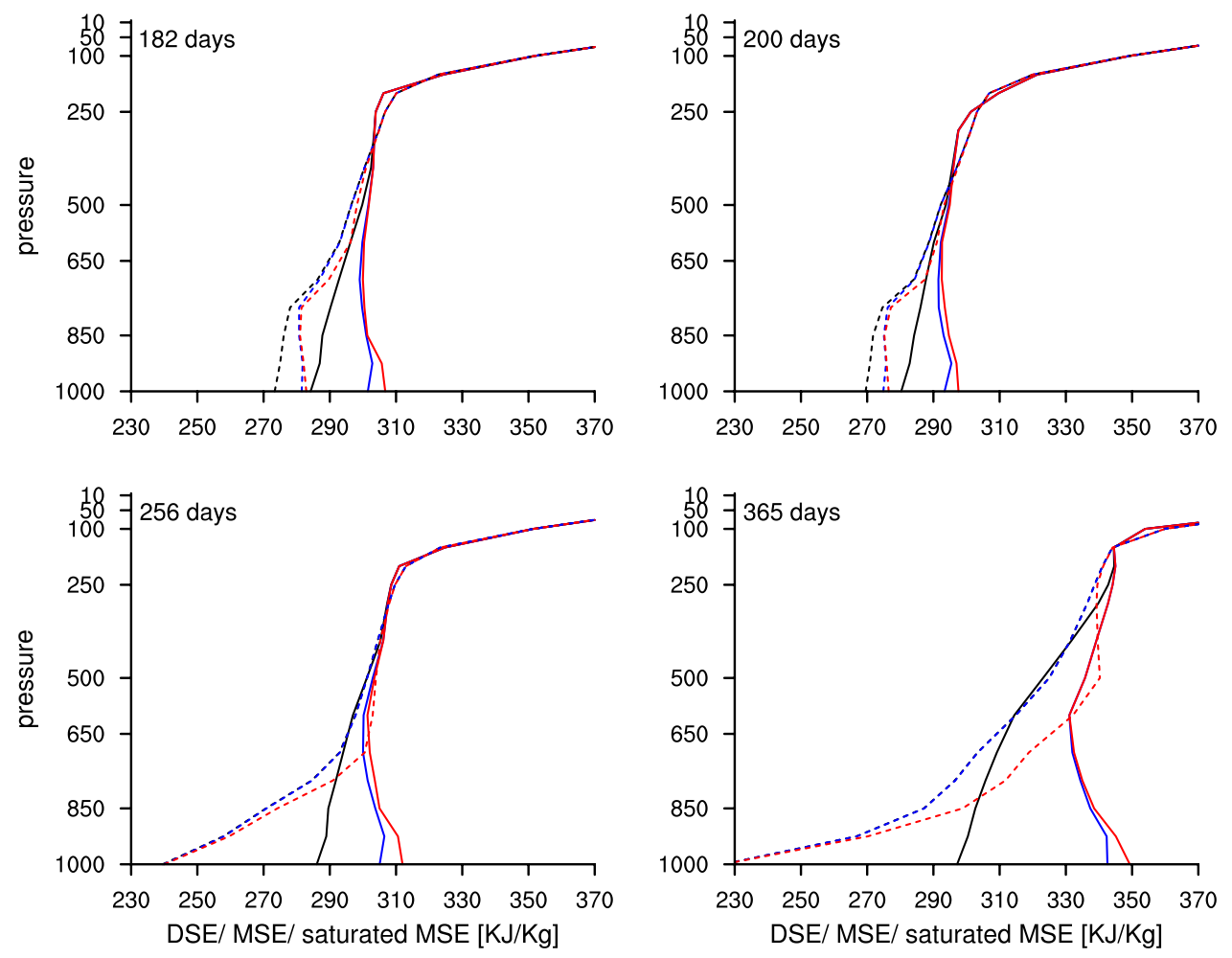

for longer periods (Fig. 8). This increase for longer rotation periods may be due to the decreasing eastward shift of the convective clouds from the substellar point. Since the incoming shortwave radiation at the top of the atmosphere is largest at the substellar point, the shift of convective clouds towards the substellar point is consistent with an increase in shortwave CRE. Note that the longwave CRE over the hot-spot area does not compensate for the cooling effect of these convective clouds. Therefore, clouds have a net cooling effect over the hot-spot area (Fig. 8).

\subsection{Atmospheric profile over the nightside}

The horizontal temperature differences in the free troposphere are negligible in the tropical region of present-day Earth climate, due to the weak Coriolis force near the equator. This is referred to as the weak temperature gradient (WTG) approximation (Sobel et al. 2001). In the case of slow rotations, the WTG approximation becomes valid globally, and the free-tropospheric temperatures are nearly constant at low pressure levels (only shown along the equator in Fig. 8). Because of the strong surface cooling during the night, the temperatures decrease strongly near the surface, and a strong thermal inversion appears. The magnitude of this inversion depends on $\mathrm{P}_{\text {rot }}$ (Fig. 8). This thermal inversion has already been discussed in the case of the synchronous rotation (Joshi et al. 1997; Merlis and Schneider 2010).
For relatively long rotation periods, a layer of low-level ice clouds extends over any open-water surface near the equator during the night. The longwave CRE of these nocturnal shallow clouds has a warming effect on the underlying surface. Beyond $\mathrm{P}_{r o t}=182$ Earth-days, an increase in $\mathrm{P}_{\text {rot }}$ causes sea ice to expand further towards sunset, thus covering the antistellar point for $\mathrm{P}_{r o t}=256,300$ and 365 Earth-days. There, the DSE, the MSE, and the saturated MSE increase with altitude, thus indicating a stably stratified atmosphere (Fig. 9). The atmosphere is dry, since the DSE curve overlaps with the MSE. The stable stratification and the dry conditions inhibit cloud formation over the area covered with sea ice. Even though the profiles in Fig. 9 are taken at the antistellar point, they are good representatives of profiles over a surface covered with sea ice on the nightside in general. Hence, the fractional cover of the nocturnal ice clouds decreases with increasing $\mathrm{P}_{\text {rot }}$ beyond 182 Earthdays (Fig. 10b, c), as well as the night-mean longwave CRE (Fig. 6). However, the global-mean longwave CRE remains nearly constant with increasing $\mathrm{P}_{r o t}$, because of the cancelling effects of the day- and night-mean longwave CRE.

\section{Global glaciation for different rotation periods}

The changes in surface temperature and sea-ice cover with long rotation periods suggest that rotation could have an influence on the point of global glaciation, which is the 

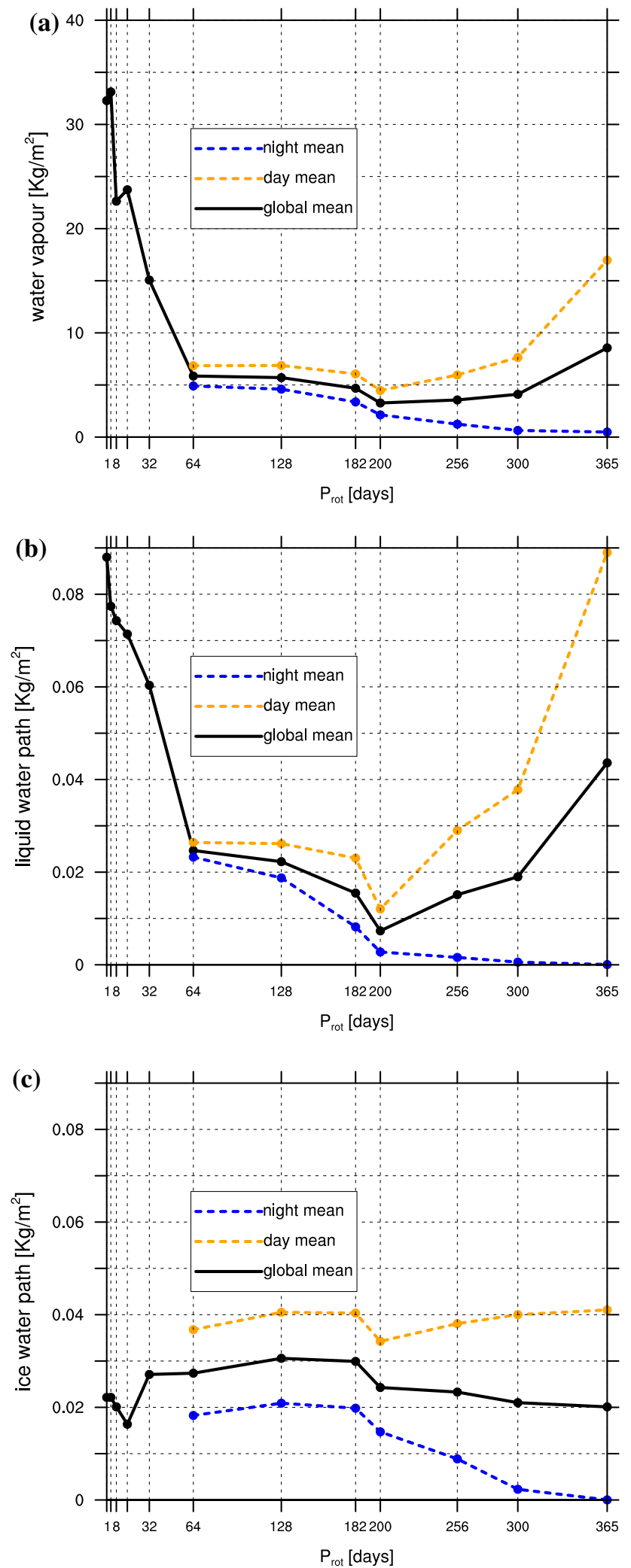

Fig. 10 Global (solid lines) and temporal mean over the last 30 years of simulation of the vertically integrated a water vapour $\left(\mathrm{kg} / \mathrm{m}^{2}\right), \mathbf{b}$ cloud water $\left(\mathrm{kg} / \mathrm{m}^{2}\right)$ and $\mathbf{c}$ cloud ice $\left(\mathrm{kg} / \mathrm{m}^{2}\right)$ as a function of $\mathrm{P}_{\text {rot }}$. The dashed yellow lines are the dayside means, the dashed blue lines are the nightside means and the solid black lines are the global means highest TSI value at which a global glaciation occurs at the surface. This is a first step towards understanding the effect of rotation on the outer edge of the habitable zone. The dependence of the inner edge of the habitable zone on the rotation period has been recently studied with a stateof-the-art model without sea ice (Yang et al. 2014). Since the climate is rather warm close to the inner edge, sea ice will likely not change their results. We perform simulations with TSI values lower than the present-day TSI (denoted by $\mathrm{TSI}_{0}$ ) for the rotation periods $\mathrm{P}_{r o t}=1,64,182,200,256$, 300 and 365 Earth-days. The highest TSI value that triggers a global glaciation $\left(\mathrm{TSI}_{g}\right.$ ) is determined for every rotation period, as well as a TSI value higher than $\mathrm{TSI}_{g}$ where the climate equilibrates without reaching a full glaciation $\left(\mathrm{TSI}_{b g}\right)$. Thus, the bifurcation point of global glaciation occurs between $\mathrm{TSI}_{b g}$ and $\mathrm{TSI}_{g}$.

For relatively fast rotations ( $\mathrm{P}_{\text {rot }}=1,64$ Earth-days), a global glaciation occurs at a TSI between 90 and $93 \%$ of $\mathrm{TSI}_{0}$, whereas non-synchronous aquaplanets with rotation periods equal to 182 and 200 Earth-days reach a global glaciation between 95 and $97 \%$ of TSI $\mathrm{T}_{0}$, and the synchronously rotating planet between 70 and $80 \%$ of TSI $_{0}$ (Fig. 11a). Hence, the exact point of global glaciation according to our simulations occurs at a TSI between TSI $_{g}$ and TSI $_{b g}$, within the shaded area in Fig. 11a. The non-synchronous aquaplanet near $\mathrm{P}_{r o t}=200$ Earth-days is the most susceptible to global glaciation, because even with $\mathrm{TSI}_{0}$ the aquaplanet with this rotation period has the largest sea-ice cover over the dayside and the lowest surface temperature and tropospheric moisture over the hot-spot. For $\mathrm{TSI}_{b g}$, the globalmean sea-ice fraction at equilibrium increases from $40 \%$ at $\mathrm{P}_{\text {rot }}=1$ up to $87 \%$ at $\mathrm{P}_{\text {rot }}=256$ Earth-days, then decreases for longer $\mathrm{P}_{\text {rot }}$ (Fig. 11b).

Therefore, slowly non-synchronously rotating planets are fully frozen at a closer distance to the star compared to planets with faster rotations and to those with synchronous rotation. A slowly non-synchronously rotating planet could then freeze over in a certain range of TSI. The rotation of this planet would slow down on geological time scales because of tidal forces exerted by other bodies. Our results suggest thus that once the planet becomes synchronously rotating, its initial state may already be in a global glaciation even though a lower TSI value is required in order to cause a global glaciation for the synchronous orbit. For a certain range of TSI, simulations of planets with synchronous rotation should be started from a state of global glaciation.

\section{Discussion}

We have demonstrated that increasing the rotation period in aquaplanet simulations leads to an increase in sea-ice extent 


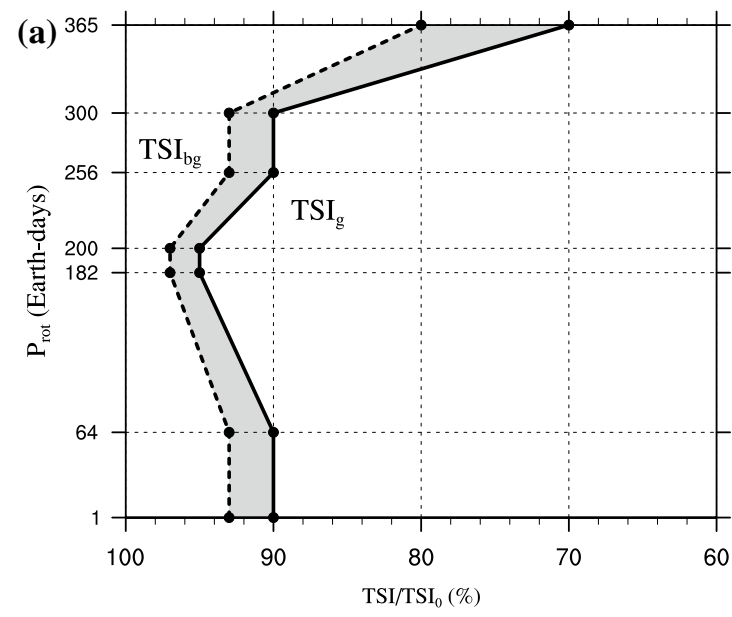

Fig. 11 a The point of global glaciation for each $\mathrm{P}_{\text {rot }}$ occurs between $\mathrm{TSI}_{b g}$ and $\mathrm{TSI}_{g}$ (in grey shade). TSI ${ }_{g}$ is the TSI value that triggers a global glaciation as a function of $\mathrm{P}_{r o t}$. $\mathrm{TSI}_{b g}$ corresponds to the simulation with a higher TSI value than TSI $_{g}$ where the equilibrium aqua-

and to a decrease in surface temperatures. However, at each $\mathrm{P}_{\text {rot }}$, the global-mean surface temperature is substantially higher when sea ice is not included in our sensitivity simulations iceOFF and in the simulations performed by Yang et al. (2014). For similar rotation periods, the GMST in our iceOFF simulations is around $10 \mathrm{~K}$ higher than the one in Yang et al. (2014) (Fig. 1). The difference in the GMST between our iceOFF simulations and Yang et al. (2014) is partly caused by the higher $\mathrm{CO}_{2}$ concentration in our study, with 800 ppmv in comparison to only 400 ppmv in Yang et al. (2014). Another factor that contributes to the difference in GMST is the global-mean planetary albedo that is higher in the simulations by Yang et al. (2014) (Fig. 2a). Nevertheless, the trends of the GMST and of the globalmean $\alpha_{P}$ as a function of $\mathrm{P}_{r o t}$ are similar in both GCMs, in ECHAM6 and in CAM3. And, since both GCMs also show that deep convective clouds form over the substellar region at slow rotations, higher confidence is attained with regard to the resulting climate at these extreme values of rotation.

The global-mean fraction of sea ice in iceON with present-day TSI are substantially larger than the values found by Way et al. (2015), where the aquaplanet simulations had a mixed-layer depth of $100 \mathrm{~m}$. The global-mean fraction of sea ice increases from $21 \%$ up to $70 \%$ with increasing the rotation period from 16 to 256 Earth-days in iceON, while it only increases from 5\% to nearly $8 \%$ in Way et al. (2015). It is also surprising that their reference simulation with a rotation period of 1 Earth-day has the highest global seaice fraction of $12 \%$, even though the global-mean surface temperature is considerably lower for slower rotations. This would imply an extremely strong atmospheric energy transport to even out the strong surface heating gradients. The GMST in Way et al. (2015) are comparable to the GMST of

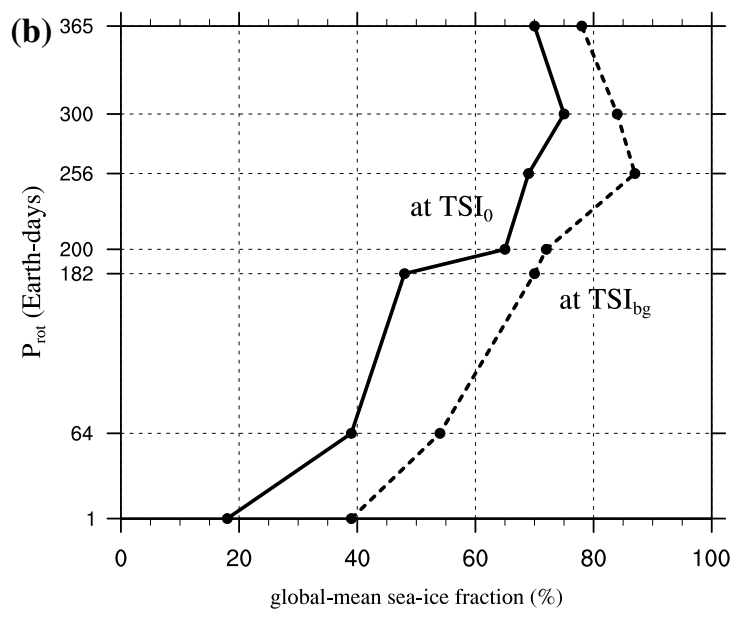

planet state does not reach full glaciation. b The global and temporal mean over the last 30 years of the sea-ice fraction (\%) as a function of $\mathrm{P}_{r o t}$ is noted for simulations with $\mathrm{TSI}_{b g}$ (dashed line) and simulations with $\mathrm{TSI}_{0}$ (solid line)

our iceOFF simulations rather than iceON, for reasons that are not clear.

The large impact of sea ice on the climate of slowly rotating aquaplanets is caused by the large contribution of the sea-ice albedo to the planetary albedo wherever sea ice persists significantly into the dayside and over low latitudes. This influence is particularly large in our simulations with $\mathrm{P}_{\text {rot }}$ between 64 and 300 Earth-days. It is, however, uncertain how far our results also apply to planets orbiting other types of stars. Depending on the type of the star, the albedo of sea ice and thus its cooling effect may be smaller or larger. M-stars emit radiation with a large contribution from the near infrared, G-stars emit strongly in the visible, and F-stars in the near ultraviolet. The snow and sea ice on hypothetical planets around these stars would then have different albedos (Joshi and Haberle 2012; Shields et al. 2013, 2014). Hence, our results cannot be directly applied to slowly rotating planets orbiting other types of stars.

Taking temporal averages with respect to the time of the day has allowed us to understand how the surface albedo and the cloud-patterns along one diurnal cycle change with the rotation period. Such information is useful when analysing visible and thermal light curves of target planets that are expected to be observed in future space missions. From these curves, the rotation period and the day length of a planet can be estimated. The variability of the visible light curve along one complete orbit indicates variations of the surface albedo under a cloud-free sky and thus could be used to estimate the rotation periods of the planet. However, in the presence of clouds and atmospheric variability, the ability of accurately retrieving the rotation period is difficult, unless the time evolution of the cloud patterns correlates with the rotation period (Palle et al. 2008). Based 
on our results, we expect that determining the rotation period from photometric light curves along one diurnal orbit would be more successful for planets with long rotation periods, because of small variability in the properties of clouds at a given time of the day.

We show the first estimates of the point of global glaciation for different slow rotation periods. The point of global glaciation of our reference simulation with the present-day rotation of Earth occurs between 90 and $93 \%$ of $\mathrm{TSI}_{0}$. This glaciation limit is equal to the limit found by Abe et al. (2011) with the CCSR/NIES model and nearly equal to the glaciation limit in the CCSM4 model that occurs between 92 and $93 \%$ of TSI $_{0}$ Shields et al. (2013). In order to determine the outer edge of the habitable zone of the aquaplanet as a function of the rotation period, a denser $\mathrm{CO}_{2}$ atmosphere should be considered because of the silicate-weathering feedback (Kasting et al. 1993; Kopparapu et al. 2014), which is expected to trap more $\mathrm{CO}_{2}$ in the atmosphere as the temperature drops. The outer edge would hence occur at the point when an additional amount of $\mathrm{CO}_{2}$ does not provide any further warming or when the $\mathrm{CO}_{2}$ condenses. Therefore, the $\mathrm{OHZ}$ is expected to occur at an orbital distance larger than the orbital distance of global glaciation. In addition to the greenhouse effect of the $\mathrm{CO}_{2}$ atmosphere, the radiative effects of $\mathrm{CO}_{2}$ ice clouds should be accounted for (Forget and Pierrehumbert 1997).

In addition to the global glaciation of the surface, the atmosphere of a planet can collapse if a major fraction of a condensable gas condensates and becomes trapped at the surface because of low temperatures. Some conditions can cause a $\mathrm{CO}_{2}$ atmosphere of a planet with synchronous (Joshi et al. 1997; Wordsworth et al. 2011) or non-synchronous rotation (Wordsworth et al. 2011) to collapse. In the case of an Earth-like atmospheric composition, the background atmosphere of $\mathrm{N}_{2}$ and $\mathrm{O}_{2}$ would only condense at extremely low temperatures. However, water vapor could collapse into permanent ice/snow traps at the surface. In our case, a collapsed state is thus equivalent to a state with global glaciation. Here, we have shown that the mass of atmospheric $\mathrm{H}_{2} \mathrm{O}$ depends on $\mathrm{P}_{\text {rot }}$ for simulations with $\mathrm{TSI}_{0}$. It decreases strongly with $\mathrm{P}_{\text {rot }}$ between 1 Earth-day and 200 Earth-days, but increases again with $\mathrm{P}_{r o t}$ beyond 200 Earthdays (Fig. 10). Furthermore, the atmosphere has collapsed in the aquaplanet simulations with different $\mathrm{P}_{r o t}$ that have reached a global glaciation at a certain TSI $_{g}$ (Fig. 11a).

Three different surface configurations are particularly often used in simulations that investigate the boundaries of the habitable zone: an aquaplanet, a land planet and a planet with Earth-like continents. These configurations have different surface properties such as the surface albedo and the distribution of water at the surface, and hence, influence the climate differently. Abe et al. (2011) found that the habitable zone of a land planet is wider than that of an aquaplanet, even under different obliquities. And a synchronously rotating land-planet exhibits a stronger diurnal contrast in surface temperature than a synchronously rotating aquaplanet (Joshi 2003). In sensitivity simulations with the orbital parameters of Venus, Yang et al. (2014) showed that the GMST in an aquaplanet configuration is $11 \mathrm{~K}$ higher than in an Earth-like continental configuration. Therefore, our results could be sensitive to the surface configuration chosen. Note that the climate of (slowly rotating) planets also depends on several fixed parameters, like the obliquity, the eccentricity, the orbital rotation period, the $\mathrm{CO}_{2}$ concentration, and the heat capacity of the mixed layer. Performing sensitivity studies with respect to each of the mentioned parameters is, however, beyond the scope of our study.

In order to overcome the limitations of a fixed depth mixed-layer ocean, Hu and Yang (2014) have performed a full atmosphere-ocean coupled aquaplanet simulation with the orbital parameters of the tidally-locked planet Gliese 581g. The zonal oceanic heat transport caused by the dynamical ocean circulation was found to reduce the seaice thickness on the nightside to $5 \mathrm{~m}$ (Hu and Yang 2014) compared to a sea-ice thickness of $100 \mathrm{~m}$ in the uncoupled simulations (Pierrehumbert 2010). However, their synchronous rotation period is 36.7 Earth-days, while ours is 365 Earth-days. We therefore expect a smaller day-tonight oceanic heat transport in our case because the geostrophic balance breaks down at longer rotation period, and thus the ocean currents won't be necessarily zonal as in Hu and Yang (2014). Nonetheless, we expect that ocean circulation would reduce the temperature gradients and the sea-ice cover for non-synchronous rotations. Surprisingly, Way et al. (2015) find the opposite, where the global-mean sea-ice cover is higher when their atmosphere is coupled to a dynamical ocean (Way et al. 2015). Their simulation with $\mathrm{P}_{r o t}=1$ Earth-day surprisingly shows a global-mean sea-ice cover (55\%) that is larger than any simulation with a longer rotation period. This highlight that the oceanic heat transport is difficult to predict without good knowledge of the details of the setup and that oceanic circulation regimes may be strongly model-dependent. Note that for presentday rotation, the mean climate of a fully-coupled aquaplanet strongly differs between various GCMs (Smith et al. 2006; Marshall et al. 2007). Overall, it may be interesting to investigate non-synchronous rotation periods in a fully coupled setup, especially since the sea-ice cover would depend on the underlying ocean circulation.

\section{Conclusion}

We have investigated the influence of sea ice on the mean climate of a slowly rotating aquaplanet, by performing 
simulations for a variety of different rotation periods ranging from one Earth-day to 365 Earth-days (in which case the planet is synchronously rotating). A large contribution to the cooling of the climate with increasing $\mathrm{P}_{\text {rot }}$ comes from the increase in planetary albedo. The high planetary albedo at long rotation periods is caused partly by the deep convective clouds over the substellar region and partly by the high surface albedo of sea ice that extends to low latitudes in the morning. The latter contribution could not be identified in previous studies where sea ice was not accounted for. Clouds have a net cooling effect on the climate at any rotation period. However, the cooling is significantly weaker in simulations with non-synchronous rotations than in the synchronous case. Moreover, clouds act to smooth out the diurnal differences by strongly cooling during the day and weakly warming during the night.

We showed the first estimates of the highest TSI leading to a global glaciation as a function of the rotation period. The point of global glaciation of slowly non-synchronously rotating planets is closer to the star than for faster rotations, and it is the most distant from the star in the synchronous case. Therefore, we propose to include the rotation period when investigating the limits of the habitable zone of a planet, and to account for sea ice and its albedo in the case of planets with long rotation periods.

Acknowledgements Open access funding provided by Max Planck Society. We thank Thorsten Mauritsen for a thorough internal review and many valuable comments. We thank Jun Yang for sharing the data from CAM4. We thank Dorian Abbot and the two anonymous reviewers for their helpful and constructive comments on the manuscript. We thank the Max Planck Society for the Advancement of Science for financial support. Computational resources were made available by Deutsches Klimarechenzentrum (DKRZ). Primary data and scripts used in the analysis and other supplementary information that may be useful in reproducing the author's work are archived by the Max Planck Institute for Meteorology and can be obtained by contacting publications@mpimet.mpg.de.

Open Access This article is distributed under the terms of the Creative Commons Attribution 4.0 International License (http:// creativecommons.org/licenses/by/4.0/), which permits unrestricted use, distribution, and reproduction in any medium, provided you give appropriate credit to the original author(s) and the source, provide a link to the Creative Commons license, and indicate if changes were made.

\section{References}

Abe Y, Abe-Ouchi A, Sleep NH, Zahnle KJ (2011) Habitable zone limits for dry planets. Astrobiology 11(5):443-460

Bechtold P, Semane N, Lopez P, Chaboureau J-P, Beljaars A, Bormann N (2014) Representing equilibrium and nonequilibrium convection in large-scale models. J Atmos Sci 71(2):734-753

Bony S, Dufresne J-L, Le Treut H, Morcrette J-J, Senior C (2004) On dynamic and thermodynamic components of cloud changes. Clim Dyn 22(2-3):71-86
Del Genio A, Zhou W (1996) Simulations of superrotation on slowly rotating planets: sensitivity to rotation and initial condition. Icarus 120(2):332-343

Donohoe A, Battisti DS (2011) Atmospheric and surface contributions to planetary albedo. J Clim 24(16):4402-4418

Edson A, Lee S, Bannon P, Kasting JF, Pollard D (2011) Atmospheric circulations of terrestrial planets orbiting low-mass stars. Icarus 212(1):1-13

Forget F, Pierrehumbert RT (1997) Warming early Mars with carbon dioxide clouds that scatter infrared radiation. Science (NY) 278(5341):1273-1276

Hu Y, Yang J (2014) Role of ocean heat transport in climates of tidally locked exoplanets around $\mathrm{M}$ dwarf stars. Proc Natl Acad Sci USA 111(2):629-634

Hunt BG (1979) The influence of the Earth's rotation rate on the general circulation of the atmosphere. J Atmos Sci 36(8):1392-1408

Iacono MJ, Delamere JS, Mlawer EJ, Shephard MW, Clough SA, Collins WD (2008) Radiative forcing by long-lived greenhouse gases: calculations with the AER radiative transfer models. J Geophys Res 113(13):2-9

Joshi M (2003) Climate model studies of synchronously rotating planets. Astrobiology 3(2):415-427

Joshi MM, Haberle RM (2012) Suppression of the water ice and snow albedo feedback on planets orbiting Red Dwarf stars and the subsequent widening of the habitable zone. Astrobiology 12(1):3-8

Joshi MM, Haberle RM, Reynolds RT (1997) Simulations of the atmospheres of synchronously rotating terrestrial planets orbiting M Dwarfs: conditions for atmospheric collapse and the implications for habitability. Icarus 129:450-465

Kaspi Y, Showman AP (2015) Atmospheric dynamics of terrestrial exoplanets over a wide range of orbital and atmospheric parameters. Astrophys J 804(1):60

Kasting JF, Whitmire DP, Reynolds RT (1993) Habitable zones around main sequence stars. Icarus 101:108-128

Koll DDB, Abbot DS (2015) Deciphering thermal phase curves of dry, tidally locked terrestrial planets. Astrophys J 802(1):21

Kopparapu RK, Ramirez R, Kasting JF, Eymet V, Robinson TD, Mahadevan S, Terrien RC, Domagal-Goldman S, Meadows V, Deshpande R (2013) Habitable zones around main-sequence stars: new estimates. Astrophys J 765(2):131

Kopparapu RK, Ramirez RM, SchottelKotte J, Kasting JF, DomagalGoldman S, Eymet V (2014) Habitable zones around mainsequence stars: dependence on planetary mass. Astrophys J 787(2):L29

Leconte J, Forget F, Charnay B, Wordsworth R, Pottier A (2013) Increased insolation threshold for runaway greenhouse processes on Earth-like planets. Nature 504(7479):268-271

Leconte J, Wu H, Menou K, Murray N (2015) Asynchronous rotation of Earth-mass planets in the habitable zone of lower-mass stars. Science 347(6222):632-635

Lohmann U, Roeckner E (1996) Design and performance of a new cloud microphysics scheme developed for the ECHAM general circulation model. Clim Dyn 12(8):557-572

Makarov VV, Efroimsky M (2013) No pseudosynchronous rotation for terrestrial planets and moons. Astrophys J 764(1):27

Makarov VV, Berghea C, Efroimsky M (2012) Dynamical evolution and spin-orbit resonances of potentially habitable exoplanets: the case of GJ $581 \mathrm{~d}$. Astrophys J 761:83

Marshall J, Ferreira D, Campin J-M, Enderton D (2007) Mean climate and variability of the atmosphere and ocean on an aquaplanet. $\mathrm{J}$ Atmos Sci 64:4270-4286

Merlis TM, Schneider T (2010) Atmospheric dynamics of Earth-like tidally locked aquaplanets. J Adv Model Earth Syst 2(4):1-17

Mlawer EJ, Taubman SJ, Brown PD, Iacono MJ, Clough SA (1997) Radiative transfer for inhomogeneous atmospheres: RRTM, a 
validated correlated-k model for the longwave. J Geophys Res 102(D14): 16663

Navarra A, Boccaletti G (2002) Numerical general circulation experiments of sensitivity to Earth rotation rate. Clim Dyn 19(5-6):467-483

Nordeng T-E (1994) Extended versions of the convective parametrization scheme at ecmwf and their impact on the mean and transient activity of the model in the tropics. Technical report, ECMWF (European Centre for Medium-Range Weather Forcasts), Shinfield Park, Reading, RG2 9AX, UK

Palle E, Ford EB, Seager S, Montanes-Rodriguez P, Vazquez M (2008) Identifying the rotation rate and the presence of dynamic weather on extrasolar Earth-like planets from photometric observations. Astrophys J 676:1319-1329

Pierrehumbert RT (2010) A palette of climates for Gliese 581g. Astrophys J 726(1):L8

Ramanathan V, Inamdar A (2006) The radiative forcing due to clouds and water vapor. In: Kiehl JT, Ramanathan V (eds) Frontiers of Climate Modeling. Cambridge University Press, Cambridge, pp 119-151

Semtner AJ (1976) A model for the thermodynamic growth of sea ice in numerical investigations of climate. J Phys Oceanogr 6(3):379-389

Shields AL, Meadows VS, Bitz CM, Pierrehumbert RT, Joshi MM, Robinson TD (2013) The effect of host star spectral energy distribution and ice-albedo feedback on the climate of extrasolar planets. Astrobiology 13(8):715-739

Shields AL, Bitz CM, Meadows VS, Joshi MM, Robinson TD (2014) Spectrum-driven planetary deglaciation due to increases in stellar luminosity. Astrophys J 785(1):L9

Showman AP, Wordsworth RD, Merlis TM, Kaspi Y (2013) Atmospheric circulation of terrestrial exoplanets. In: Mackwell SJl et al., (ed) Comparative climatology of terrestrial planets, Univ. Arizona Press, Arizona, pp 277-326

Smith RS, Dubois C, Marotzke J (2006) Global climate and ocean circulation on an aquaplanet ocean-atmosphere general circulation model. J Clim 19:4719-4737

Sobel AH, Nilsson J, Polvani LM (2001) The weak temperature gradient approximation and balanced tropical moisture waves. J Atmos Sci 58(23):3650-3665

Stevens B, Giorgetta M, Esch M, Mauritsen T, Crueger T, Rast S, Salzmann M, Schmidt H, Bader J, Block K, Brokopf R, Fast I, Kinne S, Kornblueh L, Lohmann U, Pincus R, Reichler T, Roeckner E (2013) Atmospheric component of the MPI-M earth system model: ECHAM6. J Adv Model Earth Syst 5(2):146-172

Sundqvist H, Berge E, Kristjánsson JE (1989) Condensation and cloud parameterization studies with a mesoscale numerical weather prediction model. Mon Weather Rev 117(8):1641-1657

Tiedtke M (1989) A comprehensive mass flux scheme for cumulus parameterization in large-scale models. Mon Weather Rev 117(8):1179-1800

Way MJ, Del Genio AD, Kelley M, Aleinov I, Clune T (2015) Exploring the inner edge of the habitablezone with fully coupled oceans. arXiv: 1511.07283

Wordsworth RD, Forget F, Selsis F, Millour E, Charnay B, Madeleine J-B (2011) Gliese 581D is the first discovered terrestrial-mass exoplanet in the habitable zone. Astrophys J 733(2):L48

Yang J, Cowan NB, Abbot DS (2013) Stabilizing cloud feedback dramatically expands the habitable zone of tidally locked planets. Astrophys J 771(2):L45

Yang J, Boué G, Fabrycky DC, Abbot DS (2014) Strong dependence of the inner edge of the habitable zone on planetary rotation rate. Astrophys J 787(1):L2 